\title{
USP6 activation in nodular fasciitis by promoter-swapping gene fusions
}

Nimesh R Patel ${ }^{1,2}$, John SA Chrisinger ${ }^{3}$, Elizabeth G Demicco ${ }^{3}$, Stephen F Sarabia ${ }^{1,2}$, Jacquelyn Reuther ${ }^{1,2}$, Erica Kumar ${ }^{3,8}$, Andre M Oliveira ${ }^{4}$, Steven D Billings ${ }^{5}$, Judith VMG Bovée ${ }^{6}$, Angshumoy Roy ${ }^{1,2}$, Alexander J Lazar ${ }^{3,7}$, Dolores H Lopez-Terrada ${ }^{1,2}$ and Wei-Lien Wang 3 ,7

${ }^{1}$ Department of Pathology, Texas Children's Hospital, Houston, TX, USA; ${ }^{2}$ Department of Pathology $\&$ Immunology, Baylor College of Medicine, Houston, TX, USA; ${ }^{3}$ Department of Pathology, The University of Texas MD Anderson Cancer Center, Houston, TX, USA; ${ }^{4}$ Department of Laboratory Medicine and Pathology, Mayo Clinic, Rochester, MN, USA; ${ }^{5}$ Department of Pathology, Cleveland Clinic, Cleveland, OH, USA;

${ }^{6}$ Department of Pathology, Leiden University Medical Center, Leiden, The Netherlands and ${ }^{7}$ Department of Translational Molecular Pathology, The University of Texas MD Anderson Cancer Center, Houston, TX, USA

Nodular fasciitis is a self-limited myofibroblastic lesion that can be misdiagnosed as a sarcoma as a result of its rapid growth, cellularity, and sometimes prominent mitotic activity. A recurrent translocation $t(17 ; 22)$ has been identified in nodular fasciitis, fusing the coding region of USP6 to the promoter region of $M Y H 9$, and resulting in increased USP6 expression. A subset of cases show USP6 rearrangement without the typical fusion variants by RT-PCR, or any MYH9 rearrangement by FISH. We sought to further characterize such tumors using molecular diagnostic assays. A novel RT-PCR assay was designed to detect the two known MYH9-USP6 fusion types in formalin-fixed paraffin-embedded and frozen tissue, and a break-apart FISH assay was designed to detect USP6 rearrangement. Twenty-six cases of nodular fasciitis diagnosed between 2002 and 2013 were retrieved from the pathology files of our institutions and were confirmed to be positive by FISH and/or RT-PCR. Seven samples showed USP6 rearrangement by FISH but were negative for MYH9-USP6 fusion by RT-PCR; these cases were subjected to a next-generation sequencing assay utilizing anchored multiplex PCR technology. This assay targets a single partner gene associated with fusions in bone and soft tissue tumors for agnostic detection of gene fusion partners. Novel fusion partners were identified in all seven cases and confirmed by RT-PCR. Structurally, all fusions consisted of the juxtaposition of the entire coding region of USP6 with the promoter of the partner gene, driving increased USP6 expression. This study confirms the neoplastic nature of nodular fasciitis, defines additional pathogenic fusion partners, and adds to the growing body of literature on USP6associated neoplasia. Given the diagnostic challenges of these tumors, molecular assays can be useful ancillary tools; however, the prevalence of promoter swapping must be recognized when interpreting results. Modern Pathology (2017) 30, 1577-1588; doi:10.1038/modpathol.2017.78; published online 28 July 2017

Nodular fasciitis is a self-limited myofibroblastic/ fibroblastic tumor, which usually presents as a small but rapidly growing mass. ${ }^{1-4}$ Nodular fasciitis can occur in any age, with peak incidence between 20

Correspondence: Current address: Dr NR Patel, MD, Department of Pathology, Rhode Island Hospital and Alpert Medical School at Brown University, 167 Point Street, Coro East 3227, Providence, RI 02903, USA.

E-mail: nimeshrp3@gmail.com

${ }^{8}$ Current address: Department of Pathology, Regional Medical Laboratory, Tulsa, OK, USA.

A preliminary analysis of this work was presented as a poster at the 2012 Annual Meeting of the United States and Canadian Academy of Pathology in Vancouver, Canada.

Received 5 April 2017; revised 10 May 2017; accepted 11 May 2017; published online 28 July 2017 and 40 years of age, and is found equally in males and females. ${ }^{4,5}$ It is most commonly identified in the upper extremities, head, neck, and trunk, but can be seen almost anywhere, 6,7 including superficial presentations. ${ }^{8}$ Excision is typically curative and nodular fasciitis may spontaneously regress over time in the absence of treatment. ${ }^{1-3,9-12}$

Histologically, these tumors are composed of plump fibroblasts and myofibroblasts ${ }^{13}$ that show a 'tissue culture' appearance ${ }^{2}$ and are arranged in short, irregular bundles and fascicles. The cells contain oval nuclei with prominent nucleoli,, 2 but there is typically lack of pleomorphism/nuclear atypia and necrosis. ${ }^{1}$ The overall appearance can vary, as these tumors can be cellular, hyalinized, or show a variably myxoid matrix. ${ }^{1-4,14}$ In addition, 
microcystic change, erythrocyte extravasation, lymphocytes, histiocytes, and multinucleated giant cells may be seen. ${ }^{1-3}$ Because of its rapid growth, cellularity, prominent mitotic activity, ${ }^{2}$ and locally infiltrative growth pattern, ${ }^{1,3}$ nodular fasciitis can be misdiagnosed as a sarcoma. ${ }^{6,15}$

Previously, nodular fasciitis was speculated to be a reactive process because $10-15 \%$ of patients report a history of trauma. ${ }^{14}$ However, Erickson-Johnson et $a 1^{16}$ identified a balanced rearrangement involving the USP6 gene in 44/48 cases, supporting the idea that nodular fasciitis represents a transient, often self-resolving neoplasm driven by overexpression of USP6. 5' RACE PCR and reverse transcription-PCR (RT-PCR) identified two fusion transcript types involving MYH9 exon 1 and either USP6 exon 1 or exon 2. However, break-apart fluorescence in situ hybridization (FISH) for MYH9 showed balanced rearrangement in only $31 / 48$ cases. Additional studies using FISH for USP6 rearrangement have shown a sensitivity of $83-93 \%{ }^{17-20}$ and a specificity of $100 \% .{ }^{18,20}$ In contrast, RT-PCR yielded only $53-$ $74 \%$ sensitivity. ${ }^{17,19}$ These findings suggest that other gene partners may be involved in a subset of these tumors.

We examined a large series of nodular fasciitis cases to characterize alternative fusion genes and transcript variants. Samples were initially screened by FISH and RT-PCR. We then used a next-generation sequencing assay, the FusionPlex Sarcoma Panel (ArcherDx, Boulder, CO, USA), to identify novel fusion events in these samples. This assay performs targeted RNA sequencing using anchored multiplex PCR, which utilizes unidirectional genespecific primers to detect fusion genes by targeting one of the fusion partners-USP6 for this present series. $^{21}$ Anchored multiplex PCR technology is particularly suitable for the identification of fusion genes when there is alternative splicing and the possibility of multiple partner genes, as in the case of nodular fasciitis. The FusionPlex Sarcoma Panel targets 127 exons in 26 genes for agnostic detection of gene fusions without a priori knowledge of the partner gene. We sought to determine whether this assay would detect fusion transcripts not identified by our RT-PCR assay (which is focused on the known MYH9-USP6 fusion transcripts).

\section{Materials and methods}

\section{Tumor Samples}

With institutional review board approval, we searched the pathology archives of three institutions (University of Texas MD Anderson Cancer Center, Texas Children's Hospital, and Leiden University) and identified 26 cases of nodular fasciitis diagnosed between 2002 and 2013, with available formalinfixed, paraffin-embedded or frozen (case 5) tissue (Table 1). Cases were reviewed by bone and soft tissue pathologists (WLW, AJL, ED, SB, and JVMGB). Patient age and sex, tumor location, and available clinical information were obtained. One case (case 15) had histological features in keeping with nodular fasciitis but was located in the hand/finger and had a differential diagnosis of cellular fibroma. Seven cases were previously published in a study on superficial/dermal nodular fasciitis, ${ }^{8}$ but were further characterized and re-analyzed for this present study.

\section{USP6 FISH}

Break-apart FISH was performed on interphase nuclei for detection of USP6 rearrangement. A customized probe was developed using direct-labeled bacterial artificial chromosome DNA clones, as previously described. ${ }^{8}$ Briefly, clones RP11-794K10 and RP11-124C16 (telomeric to USP6) were both labeled using SpectrumGreen dUTP (Abbott Molecular; Abbott Park, IL, USA). Clones RP11-1022O13 and RP11-457I18 (centromeric to USP6) were labeled with SpectrumOrange dUTP (Abbott Molecular). All bacterial artificial chromosome clones were obtained from the BAC-PAC Resource at Children's Hospital Oakland Research Institute (http://bacpac.chori.org/).

The probe mixture consisted of $0.5 \mu \mathrm{l}$ of each labeled DNA combined with $1 \mu \mathrm{l}$ of nuclease-free water and $5 \mu \mathrm{l}$ LSI/WCP Hybridization Buffer (Abbott Molecular). Four-micrometer-thick tumor sections were prepared for hybridization using the Paraffin Pretreatment Reagent Kit II (Abbott Molecular), per manufacturer recommendations; however, protease treatment was increased to $32 \mathrm{~min}$. Hybridization was performed at $37^{\circ} \mathrm{C}$ for up to $72 \mathrm{~h}$ in a ThermoBrite System (Abbott Molecular). Post hybridization, slides were washed in $2 \times$ salinesodium citrate buffer (SSC) with $0.3 \%$ IGEPAL at $70^{\circ}$ $\mathrm{C}$ for $2 \mathrm{~min}$, followed by two additional washes with $2 \times$ SSC with $0.3 \%$ IGEPAL at room temperature for $1 \mathrm{~min}$ each. Vectashield Mounting Media with 4,6-diamino-2-phenylindole (Vector Laboratories, Burlingame, CA, USA) was used for counter stain and for anti-fading effects. FISH analysis was performed with a multi-filtered fluorescence microscope (Olympus BX51: Olympus; Center Valley, PA, USA) and the CytoVision imaging system (version 7.4, Leica Microsystems Inc.; Buffalo Grove, IL, USA), following standard procedures. FISH signals were scored by two pathologists (DL-T and NRP), and 200 interphase nuclei were scored for fused or split orange and green signals. Cases in which $20 \%$ of interphase nuclei had split signals were considered positive for USP6 rearrangement (Figure 1d).

\section{RT-PCR for MYH9-USP6 Fusion Transcript}

RT-PCR was performed as previously described. ${ }^{8}$ Briefly, total RNA was extracted from formalin-fixed, 
Table 1 Clinical-pathologic features

\begin{tabular}{|c|c|c|c|c|c|}
\hline Case no. & Age & Sex & Location & Largest dimension $(\mathrm{cm})$ & Additional history \\
\hline 1 & 41 & Male & Psoas & 11.0 & Lumbar pain $\times 2$ months \\
\hline 2 & 14 & Male & Cheek & 2.0 & \\
\hline 3 & 42 & Female & Arm & 2.2 & \\
\hline 4 & 12 & Female & Left axilla & 3.0 & \\
\hline 5 & 13 & Male & Left upper arm & 3.1 & Painless mass $\times 6$ weeks \\
\hline 6 & 34 & Female & Left nasolabial fold & 1.0 & $\begin{array}{l}\text { Painless mass } \times 3 \text { months (history of radiation to } \\
\text { right side of face as infant for hemangioma) }\end{array}$ \\
\hline 7 & 18 & Male & Right arm & 3.3 & Painless mass $\times 3$ weeks \\
\hline 8 & 46 & Male & Right hand & 2.0 & Slow-growing painless mass $\times 2$ months \\
\hline 9 & 27 & Male & Back & 3.0 & \\
\hline 10 & 31 & Female & Right buccal & 2.0 & Painless mass $\times 6$ weeks \\
\hline 11 & 65 & Male & Right anti-tragus & 1.0 & Painless mass $\times 2$ months \\
\hline 12 & 19 & Male & Left parotid gland & 2.1 & Neck mass $\times$ several months \\
\hline 13 & 21 & Female & Neck & 2.5 & \\
\hline 14 & 38 & Male & Left subscapula & 4.0 & $\begin{array}{l}\text { Incidental mass in patient followed for lymphoma, } \\
\text { found to be enlarging over } 2 \text { months }\end{array}$ \\
\hline 15 & 55 & Male & $\begin{array}{l}\text { Left hand, third to fourth } \\
\text { tendons }\end{array}$ & 3.0 & $\begin{array}{l}\text { Numbness and tingling in hand for } 3 \text { months, } \\
\text { decreased range of motion }\end{array}$ \\
\hline 16 & 45 & Female & Orbit & 1.5 & \\
\hline 17 & 19 & Male & Skin, right lower back & N/A & \\
\hline 18 & 60 & Male & Skin, right back & N/A & \\
\hline 19 & N/A & N/A & N/A & N/A & \\
\hline 20 & N/A & N/A & N/A & N/A & \\
\hline 21 & 6 & Male & Left arm & 2.0 & \\
\hline 22 & 12 & Female & Soft tissue, submandibular & 1.0 & \\
\hline 23 & 28 & Male & Right palm & 5.5 & Enlarging mass $\times 6$ months, intermittent pain \\
\hline 24 & 51 & Male & Pelvic lymph node & 2.2 & $\begin{array}{l}\text { History of lymphoma, stem cell transplant; } \\
\text { restaging revealed PET-avid inguinal adenopathy }\end{array}$ \\
\hline 25 & 19 & Male & Right pelvis & 5.2 & $\begin{array}{l}\text { Incidental mass found in patient presenting with } \\
\text { abdominal pain, nausea, and vomiting }\end{array}$ \\
\hline 26 & 40 & Female & Right palm & 3.1 & Sharp pain after mopping, mass $\times 1$ month \\
\hline
\end{tabular}

N/A: not available.

paraffin-embedded tissue scrolls using the Ambion RecoverAll Total Nucleic Acid Isolation Kit (Ambion, Life Technologies, ThermoFisher; Austin, TX, USA), as per the manufacturer's instructions. Reverse transcription of RNA to cDNA was performed using the Invitrogen Super Script III Reverse Transcriptase Kit (Invitrogen, Life Technologies; Carlsbad, CA, USA), according to the manufacturer's instructions, with random hexamers as primers. One forward primer (MYH9-For: 5'-GCACGGAAGGCT AAGCAAG-3') and two reverse primers (USP6-RS: 5'-GGATGTGGATGTGAACTGCG-3' and USP6-RL: 5'-CGGTGTCCCTTGTCATACTTC-3') were used. Primers were designed to detect the two known fusion types ${ }^{16}$ (Figure 1a-c). PCR was performed with an annealing temperature of $55^{\circ} \mathrm{C}$.

PCR products were resolved on $2 \%$ agarose gel by electrophoresis with ethidium bromide staining for visualization. Bidirectional Sanger sequencing of the PCR products was performed to confirm amplicon identity. Analysis was performed using web-based alignment tools and gene databases (http://blast. ncbi.nlm.nih.gov/Blast.cgi, http://www.ncbi.nlm. nih.gov/gene) with mRNA reference sequences for MYH9 (NM_002473.5) and USP6 (NM_004505.3).

Amplification of the housekeeping gene beta-actin was used as an RNA integrity control. Positive controls for USP6-MYH9 fusion consisted of clonally amplified plasmids derived from sequenceconfirmed cDNA from positive patient samples. In addition, negative controls were tested with each set of reactions.

\section{Targeted RNA Sequencing by Anchored Multiplex PCR}

Total RNA was extracted from nine formalin-fixed, paraffin-embedded nodular fasciitis specimens and quantified using the Qubit RNA assay (ThermoFisher Scientific; Waltham, MA, USA). Next-generation sequencing libraries were prepared with anchored multiplex PCR-based methodology as previously described, ${ }^{21}$ using the FusionPlex Sarcoma Panel. Libraries were generated according to the manufacturer's protocol using $100 \mathrm{ng}$ of total RNA. Amplifiable cDNA quality was assessed using the PreSeq RNA QC assay. Final libraries were quantified using the KAPA Library Quantification Kit (Kapa Biosystems, Roche; Wilmington, MA, USA) for Illumina Platforms. Illumina paired-end indexed libraries were sequenced 8-plex on a MiSeq $(2 \times 150 \mathrm{bp}, \mathrm{v} 2$ chemistry $)$ and data analyzed on a vendor-provided virtual-machine-based analysis pipeline with custom-developed output scripts. On average, sequencing with the Archer FusionPlex Sarcoma Panel generated a total of 1.07 million 
a

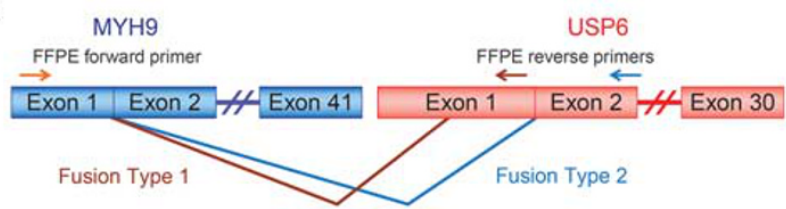

b

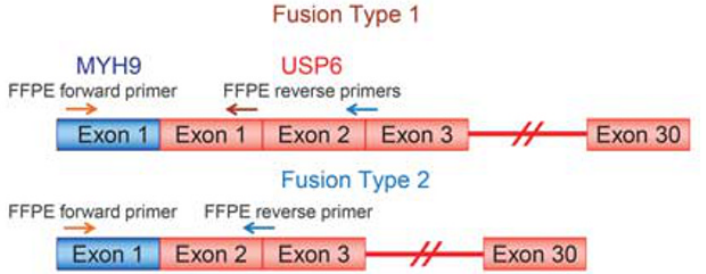

c
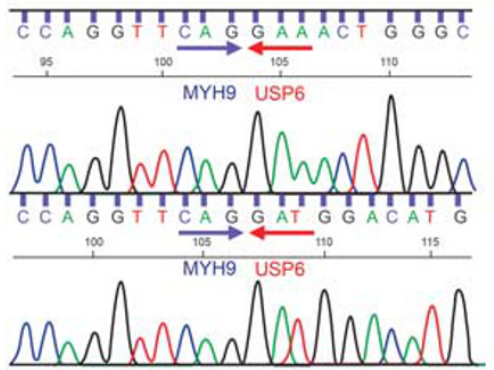

d

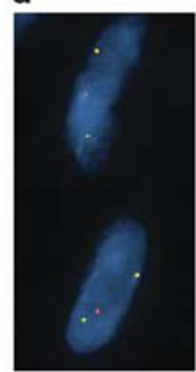

Figure 1 (a) Schematic of alternative MYH9-USP6 fusion types with corresponding design of RT-PCR primers. (b) Schematic of the resulting fusion transcripts, showing the juxtaposition of MYH9 exon 1 (encoding the 5'-UTR) to either exon 1 or 2 of USP6. Both fusion transcripts contain the entire coding sequence of USP6. Fusion type 1 may be detected with both brown (RS) and blue (RL) reverse primers, while fusion type 2 can only be detected using the blue (RL) reverse primer. (c) Case 5, electropherograms showing the type 1 (top) and type 2 (bottom) fusion transcripts with in-frame fusion of MYH9 to USP6. (d) Case 2, break-apart FISH for USP6 showing rearrangement with splitting of the green and orange signals.

paired-end reads with $\sim 28980$ unique RNA reads per sample. Sequencing quality was assessed by \% purity-filtered reads, \% bases $>$ Q30, total number of reads, total number of unique RNA reads, and percent of aligned reads with high mapping quality. Eight of nine cases yielded optimal sequencing metrics, with one case, case 10, showing low unique RNA read counts (12 751 unique RNA reads). Therefore, eight out of nine cases proceeded forward to analytic processing and fusion detection. All detected fusion genes were confirmed using RTPCR, followed by Sanger sequencing and gel electrophoresis, as described above but with specifically designed PCR primers.

\section{Results}

\section{Clinical and Pathologic Features of Samples}

Clinical and pathological parameters are summarized in Table 1. Mean age was 31.5 years (range=
6-65), with a male to female ratio of 2:1. The most common tumor sites included the upper extremities and head and neck, with additional locations including pelvis and pelvic lymph node. One case was initially diagnosed as sarcoma. Tumors ranged in size from 1.0 to $11.0 \mathrm{~cm}$, with the largest sample located within the psoas muscle (case 1). In all, 10 samples were biopsies, while 16 were excision specimens (Table 2). Histologically, the samples showed a combination of various patterns consistent with nodular fasciitis, including hypercellular, sclerotic, myxoid degeneration, and microcystic change (Figure 2). Notable cases include the $11.0 \mathrm{~cm}$ tumor, which is larger than usual for nodular fasciitis (case 1), and another case located in the tendon of the hand (case 15). In both cases, the histological findings were consistent with the diagnosis of nodular fasciitis (Figure 3). Follow-up information was available for 12 cases, with a mean follow-up time of 12.5 (1-33) months. In 10 cases, the tumor was excised without recurrence. In the remaining 2 cases, the tumor was not excised, showing regression in 1 case and lack of significant growth in the other.

\section{USP6 FISH}

FISH was successfully performed in 20 cases (Table 2), yielding positive results for USP6 locus rearrangement in all cases. Case 1 showed a variant pattern consisting of two copies of fused signals and one extra orange signal, suggestive of copy gain with an unbalanced translocation and retention of the $3^{\prime}$-portion of USP6. For the remainder of cases, testing was not performed or was not successful due to insufficient tissue or inadequate quality due to pre-analytical variables.

\section{RT-PCR for MYH9-USP6 Fusion Transcript}

RT-PCR was successfully performed in 20 cases and fusion transcripts were identified in an overlapping set of 13/20 samples (Table 2). Five cases showed only fusion type 1 , while 8 cases showed the presence of both the type 1 and type 2 transcripts, likely as a result of alternative splicing. ${ }^{16,22}$ All RTPCR-positive cases were positive by FISH, when results were available. RT-PCR was also positive in 5 cases in which there was insufficient or unavailable tissue for FISH. In contrast, 2 cases were insufficient for RT-PCR testing, but yielded positive results by FISH. Of the 7 negative RT-PCR cases, 6 were positive by FISH, while FISH was not performed in the remaining case.

\section{Targeted RNA Sequencing by Anchored Multiplex PCR}

Paired-end sequencing of target-enriched RNA libraries prepared from eight nodular fasciitis cases 
Table 2 Summary of molecular results

\begin{tabular}{|c|c|c|c|c|c|}
\hline Case no. & Specimen type & USP6 FISH & MYH9-USP6 RT-PCR & Fusion type & FusionPlex \\
\hline 1 & Excision & Positive/unbalanced & Negative & 1 & RRBP1-USP6 \\
\hline 2 & Excision & Positive & Positive & 1 & N/A \\
\hline 3 & Excision & Positive & Positive & 1 & N/A \\
\hline 4 & Excision & Positive & Positive & 1 and 2 & MYH9-USP6 \\
\hline 5 & Biopsy & Positive & Positive & 1 and 2 & N/A \\
\hline 6 & Excision & Positive & Positive & 1 and 2 & N/A \\
\hline 7 & Excision & Positive & Positive & 1 and 2 & N/A \\
\hline 8 & Excision & Positive & Positive & 1 and 2 & N/A \\
\hline 9 & Biopsy & Positive & Positive & 1 and 2 & N/A \\
\hline 10 & Biopsy & Positive & N/A & N/A & N/A \\
\hline 11 & Excision & Positive & N/A & N/A & N/A \\
\hline 12 & Excision & Positive & Negative & 3 & CALU-USP6 \\
\hline 13 & Biopsy & Positive & Negative & 1 and 2 & CTNNB1-USP6 \\
\hline 14 & Excision & Positive & Negative & 1 & MIR22HG-USP6 \\
\hline 15 & Excision & Positive & Negative & 2 & $S P A R C-U S P 6$ \\
\hline 16 & Excision & Positive & Negative & 1 and 2 & THBS2-USP6 \\
\hline 17 & Excision & Positive & $\mathrm{N} / \mathrm{A}$ & $\mathrm{N} / \mathrm{A}$ & N/A \\
\hline 18 & Biopsy & Positive & N/A & N/A & N/A \\
\hline 19 & Excision & Positive & N/A & N/A & N/A \\
\hline 20 & Biopsy & Positive & N/A & N/A & N/A \\
\hline 21 & Excision & N/A & Negative & 1 and 2 & COL6A2-USP6 \\
\hline 22 & Excision & N/A & Positive & 1 & N/A \\
\hline 23 & Biopsy & N/A & Positive & 1 & N/A \\
\hline 24 & Biopsy & N/A & Positive & 1 & N/A \\
\hline 25 & Biopsy & N/A & Positive & 1 and 2 & N/A \\
\hline 26 & Biopsy & N/A & Positive & 1 and 2 & N/A \\
\hline
\end{tabular}

N/A: not available.

using the FusionPlex Sarcoma kit yielded an average of $1.07 \times 10^{6}$ paired-end reads and 28980 unique RNA reads per sample. Analysis of FASTQ files using the Archer Analysis pipeline for fusion gene detection revealed USP6 fusions in eight of eight $(100 \%)$ cases, including a control case that was also positive by USP6 FISH and MYH9-USP6 RT-PCR. In the remaining seven cases, USP6 fusions were detected with novel 5 '-gene partners for nodular fasciitis, including RRBP1 (NM_001042576.1), CALU (NM_001219.4), CTNNB1 (NM_001098210.1), MIR22HG (NR_028502.1), SPARC (NM_003118.3), THBS2 (NM_003247.3), and COL6A2 (NM_001849.3). FusionPlex data are summarized in Table 3 . All novel fusions were confirmed using RT-PCR, followed by Sanger sequencing. RT-PCR and FusionPlex data were re-analyzed using public databases and the fusion transcripts identified in each case are summarized in Table 4.

Analysis of the novel fusion transcripts reveals that they are structurally identical to the MYH9USP6 fusion (Figure 4). Each case shows fusion of the $5^{\prime}$-UTR of the upstream gene to either exon 1 (5'-UTR) or exon 2 (one base upstream of the ATG translation initiation codon) of USP6. Some cases show multiple fusion types, which show the same USP6 fusion sites as the type 1 and type 2 MYH9 fusions (Table 4). The fusions result in promoter swap, such that the promoter and associated regulatory sequences of the 5'-partner gene are fused to the complete coding region of USP6. Activation of the $5^{\prime}$-gene promoter should therefore drive USP6 expression. In the FusionPlex assay, each of the fusion genes was supported by moderate-to-high unique split read counts (range: 92-1220 reads), with the fusion-to-wild-type fraction ranging from 20 to $100 \%$, suggestive of elevated USP6 expression from the rearranged loci.

Seven of the eight cases, including the MYH9-USP6 fusion, show inter-chromosomal rearrangements, with one case (case 14) characterized by an intrachromosomal rearrangement between MIR22HG and USP6, which are located $3.4 \mathrm{MB}$ apart on chromosome 17. The MIR22HG-USP6 gene fusion within case 14 is the only fusion identified in our study that occurs between a non-coding RNA gene and USP6. Furthermore, an additional case (case 12) has an insert within the fusion transcript, which maps to a portion of intron 1 of the $5^{\prime}$-gene $(C A L U)$. Case 1 , which was $11 \mathrm{~cm}$ in greatest dimension, showed RRBP1-USP6 fusion, confirming the diagnosis of nodular fasciitis. No significant association was identified between the fusion variants and either clinical behavior or histologic features of the tumors.

\section{Discussion}

USP6 is a hominoid-specific gene primarily expressed in testicular tissue that is located on chromosome $17 \mathrm{p} 13$ and is part of a family of deubiquitinating enzymes. ${ }^{23}$ Rearrangements involving the USP6 gene were first identified and characterized in aneurysmal bone cysts by Oliveira et $a l^{24,25}$ 
a
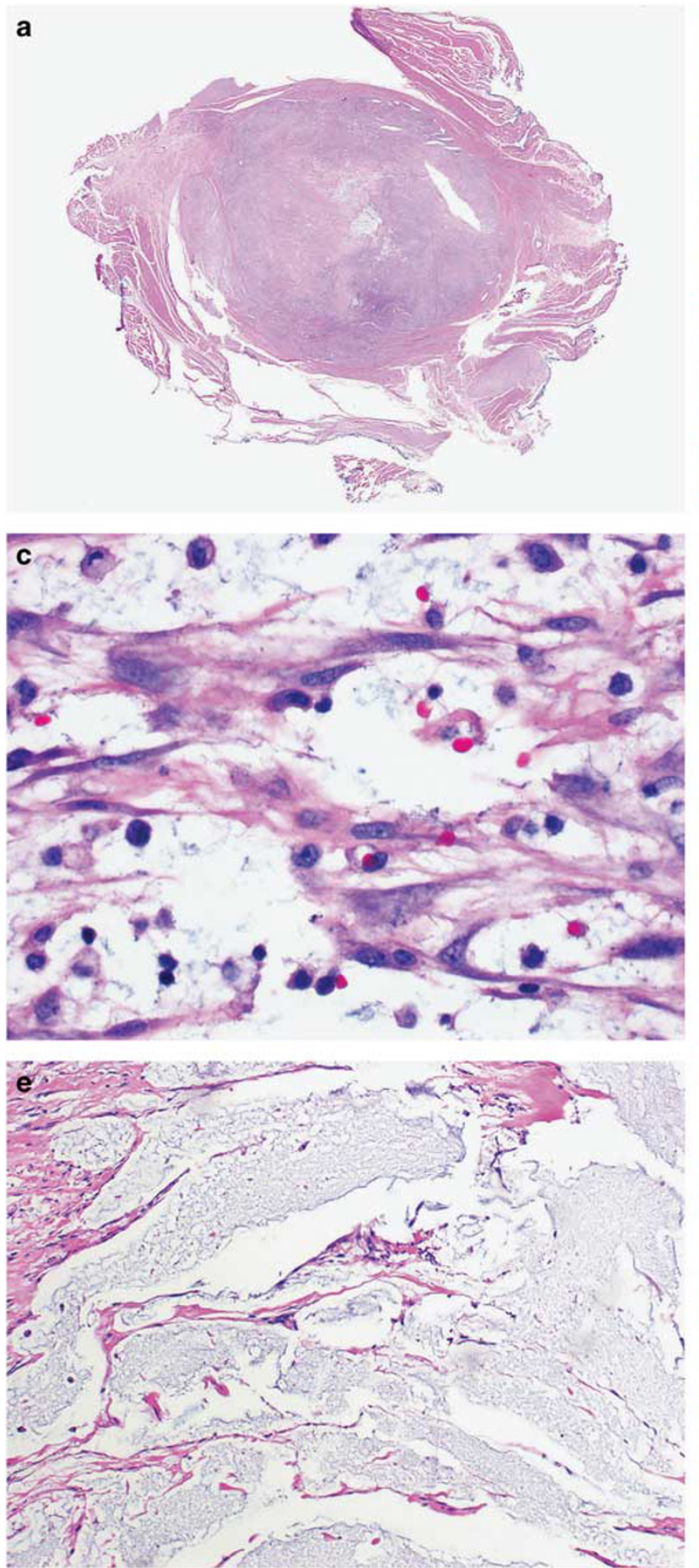
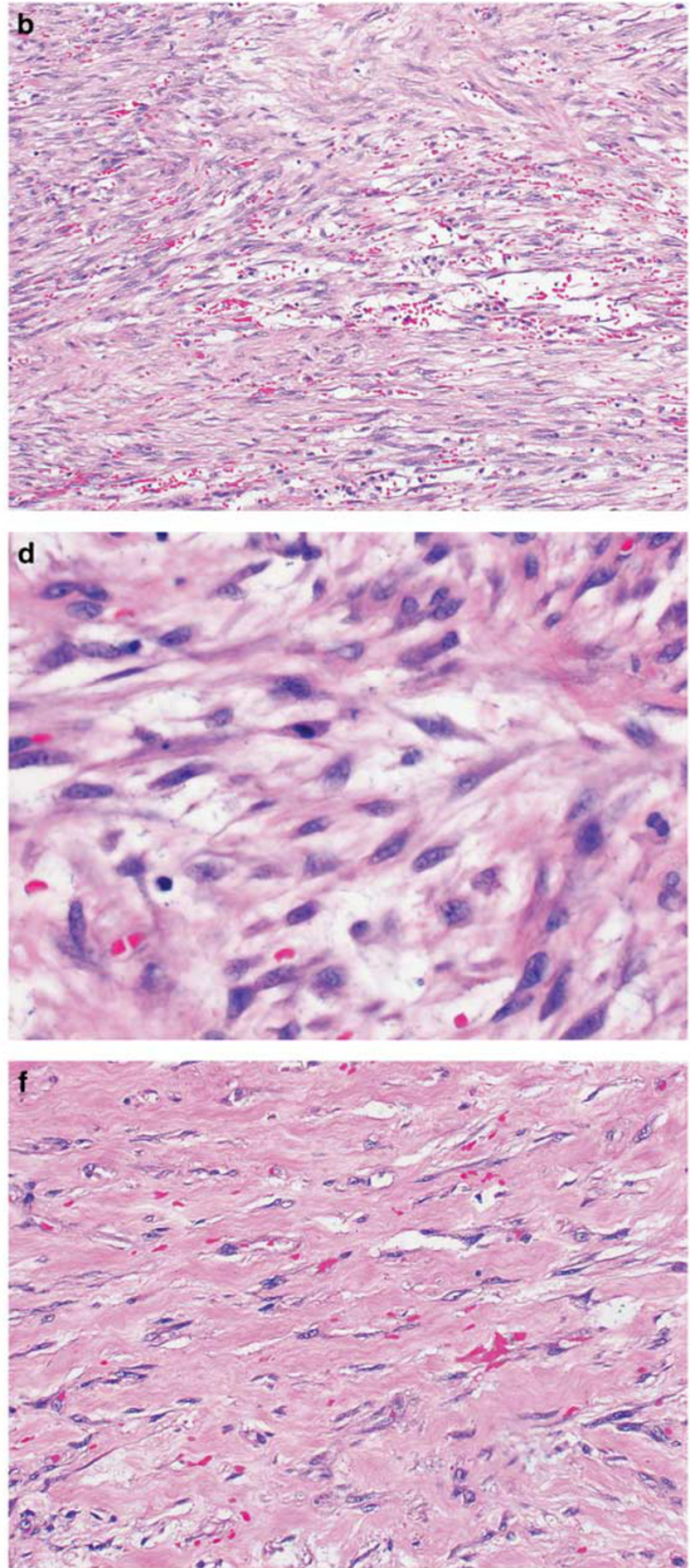

Figure 2 Representative histological features of nodular fasciitis cases seen in this series. (a) Vaguely circumscribed tumor involving skeletal muscle $(\times 1, \mathrm{H} \& \mathrm{E})$. (b) Cellular areas with tissue culture-like pattern and myxoid areas. (c) Myofibroblastic cytomorphology with extravasated red blood cells and lymphocytes. (d) Mitotic figures can be present. (e) Some tumors had prominent myxoid 'pseudocystic' areas. (f) Others had thick hyalinized bundles reminiscent of keloid collagen.

Subsequently, multiple fusion partners for USP6 were discovered in these tumors. ${ }^{26}$ Similar to nodular fasciitis, aneurysmal bone cyst is a benign tumor that grows rapidly. It was demonstrated that these tumors arise as a result of a promoter-swapping mechanism that drives transcriptional upregulation of USP6 (refs 26,27) (a mechanism of transformation seen in many tumor types). Subsequently, MYH9USP6 fusion was identified in nodular fasciitis and it was shown that increased expression of USP6 could 

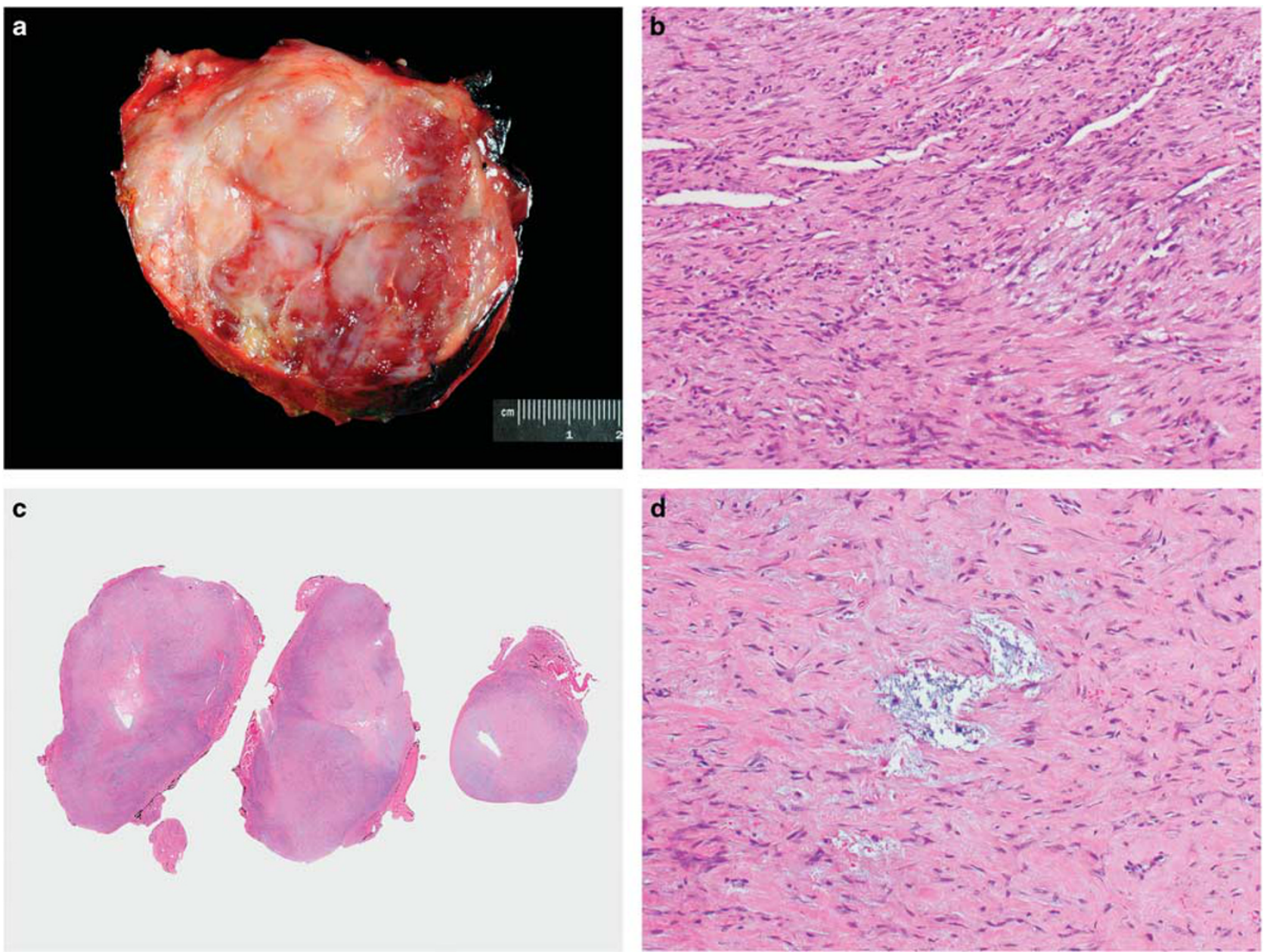

Figure 3 (a) Case 1. Cross section of nodular fasciitis, which was unusually large, measuring $11.0 \mathrm{~cm}$ in longest dimension (specimen sectioned perpendicular to longest dimension). This tumor had classic histological features (b) and was positive for RRBP1-USP6 fusion transcripts. The patient had no evidence of disease at 33 months' follow-up. (c) Case 12. Low power of a $3.0 \mathrm{~cm}$ tumor involving the tendons of the finger with histological features of nodular fasciitis (d). This tumor was found to harbor SPARC-USP6 fusion transcripts. The patient had no evidence of disease at 7 months' follow-up.

induce formation of a tumor histologically and clinically similar to nodular fasciitis in xenografts. ${ }^{16}$

In our study, FISH, RT-PCR, and targeted RNA sequencing assays were developed and utilized for the detection of USP6 rearrangement in nodular fasciitis. For clinical laboratories, several factors must be considered when implementing various methodologies to detect genomic events associated with tumors. For the detection of fusion transcripts, RT-PCR can be an ideal method when there are a limited number of fusion variants. However, RNA extracted from formalin-fixed, paraffin-embedded tissue is often fragmented to $<300$ bases in length and this fragmentation increases with storage time ${ }^{28}$ and more harsh conditions. Assays that are not appropriately designed may result in false negatives.

On the basis of the previously described fusion types, ${ }^{16}$ we designed an RT-PCR assay to detect both types 1 and 2. Several cases in our study harbored both fusion transcripts in the same sample. Alternative splicing has been well documented in fusion genes associated with bone and soft tissue tumors, ${ }^{22,29,30}$ and this likely explains the findings in our cases and in similar cases in the literature ${ }^{16}$ (Figure 1). Such an assay is highly specific, but it cannot detect other fusion partners. Moreover, if there is alternative splicing, amplicons that are larger or have different fusion sites may not be detected.

A break-apart FISH assay is especially useful when there is a single gene fused with a variety of partner genes, as in the case of aneurysmal bone cyst or, now, nodular fasciitis. USP6 rearrangement has also been identified in a variety of other tumors, including cellular fibroma of tendon sheath ${ }^{31}$ and giant cell reparative granulomas of the hands and feet (a subset of aneurysmal bone cyst), ${ }^{32}$ allowing for the implementation of a single assay across these diseases. Occasionally, unusual patterns may be identified and it can be difficult to determine the clinical significance. The pattern identified in case 1 was also described in one of six cases analyzed by Papp et $a l,{ }^{33}$ consisting of two fused signals and one extra 


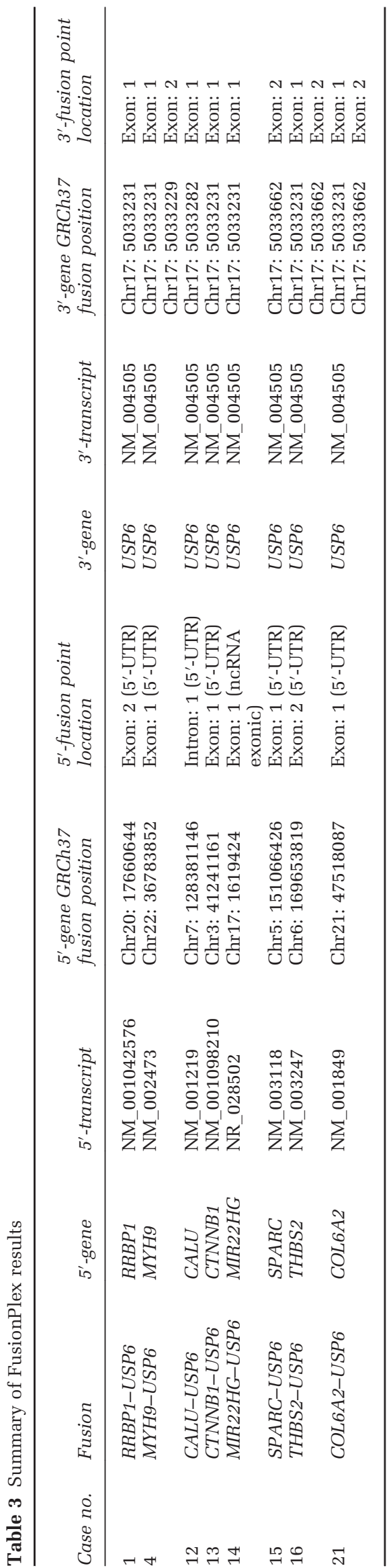

$3^{\prime}$-signal. In our experience, normal cases may show split signals in a low percentage of cells. When interpreting results, it is important to adhere to strict criteria regarding number of cells scored, distance between the orange and green signals, and validated cutoff for percentage of split cells in positive cases.

The discrepancy between FISH and RT-PCR results in our study suggested the possibility of alterative fusion types or partner genes. Alternative fusion partners have already been described in aneurysmal bone cyst and Guo et $a l^{34}$ identified PPP6R3-USP6 amplification in a case they termed 'malignant nodular fasciitis.' Anchored multiplex PCR is a powerful method for identifying alternative fusion partners, although it is not completely unbiased, as one partner gene must be targeted. During the composition of this manuscript, several other groups published studies using the Archer FusionPlex Sarcoma Panel to characterize USP6 rearrangement partners in aneurysmal bone cyst ${ }^{35}$ and cellular fibroma of tendon sheath. ${ }^{31}$

Of 26 total positive cases in our study of nodular fasciitis, 7 showed novel fusion partners, expanding the spectrum of genes known to be fused with USP6 in this tumor. In addition to encoding a ubiquitinspecific protease, the USP6 protein also contains a TBC (Tre-2/Bub2/Cdc16) domain implicated in GTPase regulation and trafficking. ${ }^{23}$ The two MYH9-USP6 fusion types show retention of both the TBC and UBP (or USP, ubiquitin-specific peptidase) domains. ${ }^{20}$ Similar to $M Y H 9$ fusion, some of the novel fusions showed alternative splicing, with the simultaneous presence of both fusion transcripts (as detected by next-generation sequencing and/or RT-PCR; Table 4). In all cases, the entire coding sequence of USP6 is retained. USP6 functions in remodeling of the cytoskeleton and extracellular matrix, inflammatory response, cell signaling, cellular trafficking, and protein turnover, ${ }^{23,27,36-44}$ but its specific role in tumorigenesis is not entirely clear. More recently, Quick et al ${ }^{45}$ reported that Jak1-STAT3 signaling has an essential role in USP6-related tumorigenesis, while Madan et $a l^{46}$ suggested a role for Wnt signaling.

In the majority of nodular fasciitis cases, USP6 fuses to $M Y H 9$, which is located on chromosome 22q12.3-q13 and encodes for a non-muscle myosin protein involved in cytokinesis, cell motility, maintenance of cell shape, and disassembly of the actin network in crawling cells. ${ }^{16}$ In the body, it is normally present at low levels, except in fibroblasts, endothelial cells, macrophages, leukocytes, and certain renal cells. Mutations have been associated with macrothrombocytopenias, ${ }^{47}$ and MYH9 has been reported to be fused to $A L K$ in a case of anaplastic large cell lymphoma. ${ }^{48}$

We identified several novel fusion partners for USP6 in nodular fasciitis. COL6A2 encodes for the collagen type VI alpha 2 chain. Mutations in COL6A2 are associated with inherited muscular dystrophies. ${ }^{49}$ Collagen VI is enriched in the 


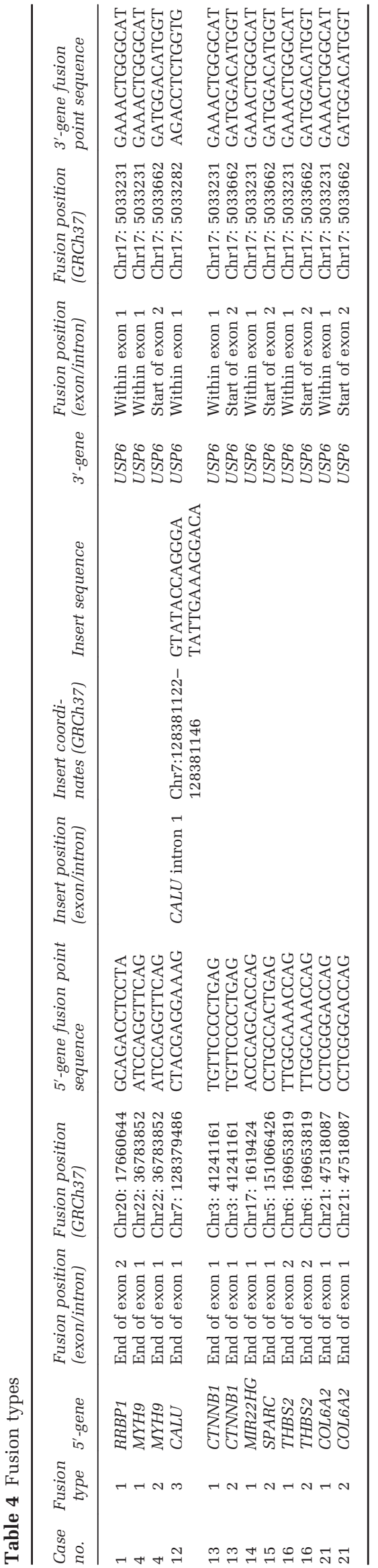

pericellular matrix of tendon fibroblasts and has an important role in tendon repair. ${ }^{49}$

THBS2 encodes for a member of the thrombospondin family of extracellular proteins, which function in cell-to-cell and cell-to-matrix communication. ${ }^{50}$ Thrombospondin-2 (TBS-2) has also been shown to inhibit angiogenesis. ${ }^{50}$ In Ewing sarcoma, the EWSR1-FLI fusion gene downregulates TBS-2. ${ }^{51}$

Case 12 showed CALU-USP6 fusion, with the fusion transcript containing an insertion that maps to CALU intron 1. The inclusion of a cryptic exonic sequence in the fusion transcript occurs commonly in other tumors, and we previously showed this is a recurrent event in epithelioid hemangioendotheliomas. ${ }^{22}$ This may be related to alterations in splice sites or splicing enhancers and silencers as a result of chromosomal translocation. ${ }^{22}$ Calumenin, encoded by $C A L U$, is a calcium-binding protein belonging to the CREC protein family. ${ }^{52}$ Different isoforms are localized to different parts of the secretory pathway, including the endoplasmic reticulum, Golgi apparatus, and extracellular medium. Calumenin is thought to have a role in inhibiting tumor metastasis, and its expression levels have been reported to be altered in cancer of the head and neck, endometrium, liver, pancreas, lung, and colon. ${ }^{53}$ CALU expression was demonstrated to be increased in the tumoral stroma (cancerassociated fibroblasts) and epithelium of colorectal cancer samples. ${ }^{54}$

Activating mutations in CTNNB1 have been associated with desmoid fibromatosis, ${ }^{55}$ indicating a role in fibroblastic tumors. CTNNB1 encodes betacatenin, which interacts with APC and has a wellcharacterized role in the Wnt signaling pathway in most cell types and cellular adhesion in epithelial cells. Furthermore, CTNNB1-PLAG1 fusion in pleomorphic adenomas of the salivary gland has been shown to result in activation of PLAG1 by a promoter-swapping mechanism..$^{56,57}$ Of note, CTNNB1-USP6 fusion was recently identified in a single case of aneurysmal bone cyst, ${ }^{35}$ another member of the biological spectrum of USP6-induced neoplasms. ${ }^{20}$

MIR22HG is a long non-coding RNA that is downregulated in lung adenocarcinoma ${ }^{58}$ and has been shown to be a responder to chemical stress, ${ }^{59}$ microgravity, ${ }^{60}$ and hypoxia. ${ }^{61}$ It serves as host gene for miR-22, which functions as a tumor suppressor by post-transcriptional regulation of p21 and represses cancer progression by inducing cellular senescence. ${ }^{60}$

SPARC encodes a cysteine-rich acidic matrixassociated protein. It is a member of a family of proteins that modulate interactions between cells and their environment by regulating growth factor signaling and extracellular matrix assembly and deposition. ${ }^{62}$ SPARC functions as both a secreted glycoprotein and an intracellular and membraneassociated protein that regulates cellular apoptotic pathways. ${ }^{62}$ It shows increased expression in 


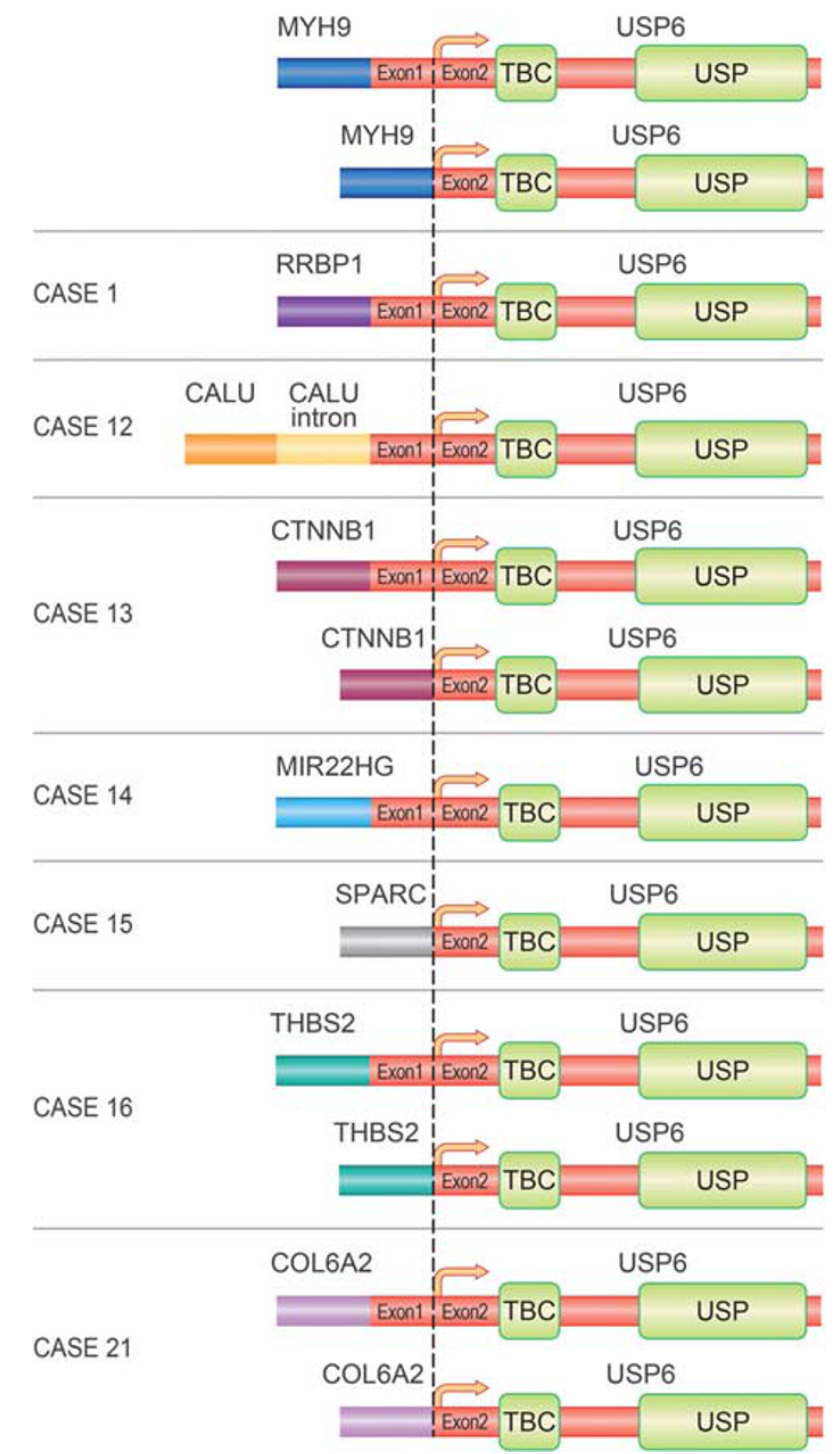

Figure 4 Schematic of fusion transcripts. The top pane shows the two MYH9-USP6 fusion types, both of which include the entire coding sequence of USP6 (transcription start site indicated by an arrow). The TBC and USP (UBP) domains of the USP6 protein are also indicated. In the remaining panes, the other novel fusion transcripts identified in this study are shown. Case 12 shows the inclusion of a cryptic exon from $C A L U$, while cases 13,16 , and 21 show the presence of two fusion transcripts, with identical USP6 fusion sites as those seen in MYH9 fusions.

epithelial cells from tissues with a high turnover rate during tissue injury and inflammation, as well as during abnormal tissue growth associated with neoplasia. ${ }^{62}$ Interestingly, this fusion was found in a case occurring in the tendon of the hand (case 15). The histological features were in keeping with nodular fasciitis, but given the location, a differential diagnosis of cellular fibroma could also be entertained. Recently, Carter et al ${ }^{31}$ described USP6 gene rearrangements in six of nine cellular fibromas of tendon sheath by FISH. No MYH9 rearrangements or MYH9-USP6 and CDH11-USP6 fusion transcripts were detected by RT-PCR assays. Given the overlapping features, they postulate that some cellular fibromas of tendon sheath may in fact be tenosynovial nodular fasciitis. Our case also highlights the similarities between these entities, both histologically and molecularly, and now with a fusion partner of SPARC.

All of the fusion partners for USP6 likely provide promoters that lead to increased transcription over what would be seen if the USP6 promoter was left in place. Some of these fused promoters could represent genes that are activated specifically within the cellular context of the myofibroblasts that comprise nodular fasciitis-a conjecture that requires further investigation.

To our knowledge, we also report the largest nodular fasciitis case with molecular confirmation in the literature. Although most cases are small, tumors up to $9 \mathrm{~cm}$ have been previously described. ${ }^{63,64}$ Case 1 in our study was $11 \mathrm{~cm}$ and showed an unusual FISH pattern with unbalanced translocation and RRBP1-USP6 fusion by nextgeneration sequencing. The association between the phenotype (ie, large size) and fusion variant is not certain; however, the patient was noted to be alive with no evidence of disease (recurrences or metastases) 10 years post excision. Ribosome-binding protein $1(R R B P 1)$ encodes for a coiled-coil protein that has a role in microtubule binding and in the interaction between the endoplasmic reticulum and ribosomes. ${ }^{65}$ RRBP1 is highly expressed in cells such as fibroblasts and is necessary for secretory activity. ${ }^{66}$ Overexpression of $R R B P 1$ is associated with poor prognosis in breast and colorectal cancer, and RRBP1-ALK fusion has been identified in several cases of epithelioid inflammatory myofibroblastic sarcoma. ${ }^{65}$

As previously mentioned, Guo et al ${ }^{34}$ described a patient who had multiply recurrent nodular fasciitis over 10 years before developing a metastasis. ${ }^{34}$ This is the first reported case of nodular fasciitis with malignant behavior, and the tumor was found to harbor a novel fusion transcript PPP6R3-USP6. This fusion was not seen in our series, and no differences were seen in the behavior and histological features in the tumors of various fusion transcripts and types.

In summary, we identified seven novel fusion partners for USP6 in nodular fasciitis, highlighting the importance of USP6 expression and promoterswapping fusions in the etiology of this neoplasm. The partner genes normally have a role in the interaction between the cell and extracellular matrix, inflammation, myo/fibroblastic activity, or stress response. The association of the coding sequence of USP6 with the promoter of these genes results in increased expression of USP6 within the milieu that gives rise to nodular fasciitis. This may account for the histologic features of inflammation and myofibroblastic proliferation associated with these tumors. Molecular testing for USP6 rearrangement can be a useful ancillary tool, though pathologists need to be cognizant of the limitations of 
each technique to detect multiple partners. Recently, a similar study examining aneurysmal bone cysts by anchored multiplex PCR expanded the spectrum of associated partner genes ${ }^{35}$ and, together, this provides additional evidence for the neoplastic nature of USP6-associated tumors. Further studies must be performed to elucidate the biology and phenomenon of transient neoplasia inherent to nodular fasciitis.

\section{Acknowledgments}

We thank Karen Prince and Kim-Anh Vu for their help with the preparation of the images, as well as Hadi Sayeed, Kayuri Patel, Vijetha Kumar, Angela Major, and E Faith Hollingsworth for assistance in experimental procedures.

\section{Disclosure/conflict of interest}

The authors declare no conflict of interest.

\section{References}

1 Price EB Jr, Silliphant WM, Shuman R. Nodular fasciitis: a clinicopathologic analysis of 65 cases. Am J Clin Pathol 1961;35:122-136.

2 Konwaler BE, Keasbey L, Kaplan L. Subcutaneous pseudosarcomatous fibromatosis (fasciitis). Am J Clin Pathol 1955;25:241-252.

3 Stout AP. Pseudosarcomatous fascitis in children. Cancer 1961;14:1216-1222.

4 Bernstein KE, Lattes R. Nodular (pseudosarcomatous) fasciitis, a nonrecurrent lesion: clinicopathologic study of 134 cases. Cancer 1982;49:1668-1678.

5 Tomita S, Thompson K, Carver $\mathrm{T}$, et al. Nodular fasciitis: a sarcomatous impersonator. J Pediatr Surg 2009;44:e17-e19.

6 Lu L, Lao IW, Liu X, et al. Nodular fasciitis: a retrospective study of 272 cases from China with clinicopathologic and radiologic correlation. Ann Diagn Pathol 2015;19:180-185.

7 Goldblum JR, Folpe AL, Weiss SW. Benign fibroblastic/ myofibroblastic proliferations, including superficial fibromatoses. In: Goldblum JR, Folpe AL, Weiss SW (eds). Enzinger and Weiss's Soft Tissue Tumors. 6th edn. Elsevier Saunders: Philadelphia, PA, USA, 2014, pp 188-255.

8 Kumar E, Patel NR, Demicco EG, et al. Cutaneous nodular fasciitis with genetic analysis: a case series. J Cutan Pathol 2016;43:1143-1149.

9 Majumdar B. Spontaneously regressing nodular fasciitis of the neck. J Laryngol Otol 1983;97:973-977.

10 Bemrich-Stolz CJ, Kelly DR, Muensterer OJ, et al. Single institution series of nodular fasciitis in children. J Pediatr Hematol Oncol 2010;32:354-357.

11 Grobmyer SR, Knapik JA, Foss RM, et al. Nodular fasciitis: differential considerations and current management strategies. Am Surg 2009;75:610-614.

12 Yanagisawa A, Okada H. Nodular fasciitis with degeneration and regression. J Craniofac Surg 2008;19: 1167-1170.
13 Eyden B, Banerjee SS, Shenjere P, et al. The myofibroblast and its tumours. J Clin Pathol 2009;62:236-249.

14 Rosenberg AE. Pseudosarcomas of soft tissue. Arch Pathol Lab Med 2008;132:579-586.

15 Arbiser ZK, Folpe AL, Weiss SW. Consultative (expert) second opinions in soft tissue pathology. Analysis of problem-prone diagnostic situations. Am J Clin Pathol 2001;116:473-476.

16 Erickson-Johnson MR, Chou MM, Evers BR, et al. Nodular fasciitis: a novel model of transient neoplasia induced by MYH9-USP6 gene fusion. Lab Invest 2011;91:1427-1433.

17 Chen J, Ye X, Li Y, et al. Chromosomal translocation involving USP6 gene in nodular fasciitis. Zhonghua Bing Li Xue Za Zhi 2014;43:533-536.

18 Shin C, Low I, Ng D, et al. USP6 gene rearrangement in nodular fasciitis and histological mimics. Histopathology 2016;69:784-791.

19 Amary MF, Ye H, Berisha F, et al. Detection of USP6 gene rearrangement in nodular fasciitis: an important diagnostic tool. Virchows Arch 2013;463:97-98.

20 Oliveira AM, Chou MM. USP6-induced neoplasms: the biologic spectrum of aneurysmal bone cyst and nodular fasciitis. Hum Pathol 2014;45:1-11.

21 Zheng Z, Liebers M, Zhelyazkova B, et al. Anchored multiplex PCR for targeted next-generation sequencing. Nat Med 2014;20:1479-1484.

22 Patel NR, Salim AA, Sayeed H, et al. Molecular characterization of epithelioid haemangioendotheliomas identifies novel WWTR1-CAMTA1 fusion variants. Histopathology 2015;67:699-708.

23 Oliveira AM, Chou MM. The TRE17/USP6 oncogene: a riddle wrapped in a mystery inside an enigma. Front Biosci (Schol Ed) 2012;4:321-334.

24 Oliveira AM, Perez-Atayde AR, Inwards CY, et al. USP6 and CDH11 oncogenes identify the neoplastic cell in primary aneurysmal bone cysts and are absent in so-called secondary aneurysmal bone cysts. Am J Pathol 2004;165:1773-1780.

25 Oliveira AM. USP6 (Tre2) fusion oncogenes in aneurysmal bone cyst. Cancer Res 2004;64:1920-1923.

26 Oliveira AM, Perez-Atayde AR, Dal Cin P, et al. Aneurysmal bone cyst variant translocations upregulate USP6 transcription by promoter swapping with the ZNF9, COL1A1, TRAP150, and OMD genes. Oncogene 2005;24:3419-3426.

27 Lau AW, Pringle LM, Quick L, et al. TRE17/ubiquitinspecific protease 6 (USP6) oncogene translocated in aneurysmal bone cyst blocks osteoblastic maturation via an autocrine mechanism involving bone morphogenetic protein dysregulation. J Biol Chem 2010;285:37111-37120.

28 Cronin M, Pho M, Dutta D, et al. Measurement of gene expression in archival paraffin-embedded tissues. Am J Pathol 2004;164:35-42.

29 Wang WL, Mayordomo E, Zhang W, et al. Detection and characterization of EWSR1/ATF1 and EWSR1/CREB1 chimeric transcripts in clear cell sarcoma (melanoma of soft parts). Mod Pathol 2009;22:1201-1209.

30 Coindre JM, Hostein I, Terrier P, et al. Diagnosis of clear cell sarcoma by real-time reverse transcriptase-polymerase chain reaction analysis of paraffin embedded tissues: clinicopathologic and molecular analysis of 44 patients from the French sarcoma group. Cancer 2006;107:1055-1064.

31 Carter JM, Wang X, Dong J, et al. USP6 genetic rearrangements in cellular fibroma of tendon sheath. Mod Pathol 2016;29:865-869. 
32 Agaram NP, LeLoarer FV, Zhang L, et al. USP6 gene rearrangements occur preferentially in giant cell reparative granulomas of the hands and feet but not in gnathic location. Hum Pathol 2014;45:1147-1152.

33 Papp G, Mihály D, Sápi Z. Unusual signal patterns of break-apart FISH probes used in the diagnosis of soft tissue sarcomas. Pathol Oncol Res 2017; 10.1007/ s12253-017-0200-z.

34 Guo R, Wang X, Chou MM, et al. PPP6R3-USP6 amplification: novel oncogenic mechanism in malignant nodular fasciitis. Genes Chromosomes Cancer 2016;55:640-649.

35 Guseva NV, Jaber O, Tanas MR, et al. Anchored multiplex PCR for targeted next-generation sequencing reveals recurrent and novel USP6 fusions and upregulation of USP6 expression in aneurysmal bone cyst. Genes Chromosomes Cancer 2017;56:266-277.

36 Masuda-Robens JM, Kutney SN, Qi H, et al. The TRE17 oncogene encodes a component of a novel effector pathway for Rho GTPases Cdc42 and Rac1 and stimulates actin remodeling. Mol Cell Biol 2003;23:2151-2161.

37 Martinu L, Masuda-Robens JM, Robertson SE, et al. The TBC (Tre-2/Bub2/Cdc16) domain protein TRE17 regulates plasma membrane-endosomal trafficking through activation of Arf6. Mol Cell Biol 2004;24:9752-9762.

38 Bizimungu C, Vandenbol M. At least two regions of the oncoprotein Tre2 are involved in its lack of GAP activity. Biochem Biophys Res Commun 2005;335: 883-890.

39 Bizimungu C, Thomas A, Brasseur R, et al. Mutational analysis of the TRE2 oncogene encoding an inactive RabGAP. Biotechnol Lett 2007;29:1927-1937.

40 Shen C, Ye Y, Robertson SE, et al. Calcium/calmodulin regulates ubiquitination of the ubiquitin-specific protease TRE17/USP6. J Biol Chem 2005;280:35967-35973.

41 Dechamps C, Bach S, Portetelle D, et al. The Tre2 oncoprotein, implicated in Ewing's sarcoma, interacts with two components of the cytoskeleton. Biotechnol Lett 2006;28:223-231.

42 Ye Y, Pringle LM, Lau AW, et al. TRE17/USP6 oncogene translocated in aneurysmal bone cyst induces matrix metalloproteinase production via activation of NF-kappaB. Oncogene 2010;29:3619-3629.

43 Pringle LM, Young R, Quick L, et al. Atypical mechanism of NF-kappaB activation by TRE17/ubiquitin-specific protease 6 (USP6) oncogene and its requirement in tumorigenesis. Oncogene 2012;31:3525-3535.

44 Funakoshi Y, Chou MM, Kanaho Y, et al. TRE17/USP6 regulates ubiquitylation and trafficking of cargo proteins that enter cells by clathrin-independent endocytosis. J Cell Sci 2014;127:4750-4761.

45 Quick L, Young R, Henrich IC, et al. Jak1-STAT3 signals are essential effectors of the USP6/TRE17 oncogene in tumorigenesis. Cancer Res 2016;76:5337-5347.

46 Madan B, Walker MP, Young R, et al. USP6 oncogene promotes Wnt signaling by deubiquitylating Frizzleds. Proc Natl Acad Sci USA 2016;113:E2945-E2954.

47 Economou M, Batzios SP, Pecci A, et al. MYH9-related disorders: report on a patient of Greek origin presenting with macroscopic hematuria and presenile cataract, caused by an R1165C mutation. J Pediatr Hematol Oncol 2012;34:412-415.

48 Lamant L, Gascoyne RD, Duplantier MM, et al. Nonmuscle myosin heavy chain (MYH9): a new partner fused to ALK in anaplastic large cell lymphoma. Genes Chromosomes Cancer 2003;37:427-432.
49 Sardone F, Santi S, Tagliavini F, et al. Collagen VI-NG2 axis in human tendon fibroblasts under conditions mimicking injury response. Matrix Biol 2016;55:90-105.

50 Lawler J. The functions of thrombospondin-1 and-2. Curr Opin Cell Biol 2000;12:634-640.

51 Potikyan G, Savene RO, Gaulden JM, et al. EWS/FLI1 regulates tumor angiogenesis in Ewing's sarcoma via suppression of thrombospondins. Cancer Res 2007;67: 6675-6684.

52 Honore B, Vorum H. The CREC family, a novel family of multiple EF-hand, low-affinity $\mathrm{Ca}(2+)$-binding proteins localised to the secretory pathway of mammalian cells. FEBS Lett 2000;466:11-18.

53 Zheng P, Wang Q, Teng J, et al. Calumenin and fibulin1 on tumor metastasis: implications for pharmacology. Pharmacol Res 2015;99:11-15.

54 Torres S, Bartolome RA, Mendes M, et al. Proteome profiling of cancer-associated fibroblasts identifies novel proinflammatory signatures and prognostic markers for colorectal cancer. Clin Cancer Res 2013;19:6006-6019.

55 Wang WL, Nero C, Pappo A et al. CTNNBI genotyping and APC screening in pediatric desmoid tumors: a proposed algorithm. Pediatr Dev Pathol 2012;15:361-367.

56 Kas K, Voz ML, Roijer E, et al. Promoter swapping between the genes for a novel zinc finger protein and beta-catenin in pleiomorphic adenomas with $t(3 ; 8)$ (p21;q12) translocations. Nat Genet 1997;15:170-174.

57 Matsuyama A, Hisaoka M, Nagao Y, et al. Aberrant PLAG1 expression in pleomorphic adenomas of the salivary gland: a molecular genetic and immunohistochemical study. Virchows Arch 2011;458:583-592.

$58 \mathrm{Li}$ DS, Ainiwaer JL, Sheyhiding I, et al. Identification of key long non-coding RNAs as competing endogenous RNAs for miRNA-mRNA in lung adenocarcinoma. Eur Rev Med Pharmacol Sci 2016;20:2285-2295.

59 Tani H, Onuma Y, Ito Y, et al. Long non-coding RNAs as surrogate indicators for chemical stress responses in human-induced pluripotent stem cells. PLoS ONE 2014;9:e106282.

60 Vidyasekar P, Shyamsunder P, Arun R, et al. Genome wide expression profiling of cancer cell lines cultured in microgravity reveals significant dysregulation of cell cycle and microRNA gene networks. PLoS ONE 2015;10:e0135958.

61 Voellenkle C, Garcia-Manteiga JM, Pedrotti S, et al. Implication of long noncoding RNAs in the endothelial cell response to hypoxia revealed by RNA-sequencing. Sci Rep 2016;6:24141.

62 Wong SL, Sukkar MB. The SPARC protein: an overview of its role in lung cancer and pulmonary fibrosis and its potential role in chronic airways disease. Br J Pharmacol 2017;174:3-14.

63 Montgomery EA, Meis JM. Nodular fasciitis. Its morphologic spectrum and immunohistochemical profile. Am J Surg Pathol 1991;15:942-948.

64 Coyle J, White LM, Dickson B, et al. MRI characteristics of nodular fasciitis of the musculoskeletal system. Skeletal Radiol 2013;42:975-982.

65 Lee JC, Li CF, Huang HY, et al. ALK oncoproteins in atypical inflammatory myofibroblastic tumours: novel RRBP1-ALK fusions in epithelioid inflammatory myofibroblastic sarcoma. J Pathol 2017;241:316-323.

66 Fry CS, Kirby TJ, Kosmac K, et al. Myogenic progenitor cells control extracellular matrix production by fibroblasts during skeletal muscle hypertrophy. Cell Stem Cell 2017;20:56-69. 